\title{
Attenuator systems - an old method to deviate rocks but a new testing method for developing a design concept
}

\author{
J Glover Global Risk Forum Davos, Switzerland \\ DC Wyllie Wyllie \& Norrish Rock Engineers Ltd., Canada \\ R Bucher Geobrugg Australia Pty Ltd, Australia
}

\begin{abstract}
Rockfall attenuator systems, also known as hybrid rockfall barriers or hanging nets, are a widely applied rockfall mitigation method. Although they are conceptually a straight forward design, to-date, a definitive design guide remains elusive. Among some of the first testing of these systems e.g. Glover et al. 2012, a boarder understanding of the key mechanics, with which they function, has been attained. For example, the process of deflecting rocks to increased ground contact and catching and tangling of the rocks in the attenuator systems has been observed. Nonetheless, these observations remain to be quantified in detail, so they could be developed into a design guide.

Addressing this need, a joint testing program is being carried out by Wyllie \& Norrish Rock Engineers Ltd. and Geobrugg North America, to measure and validate the performance of rockfall attenuator systems. The preliminary stages of full-scale testing have been conducted at the Nicolum Quarry in Hope, British Columbia, Canada. Tests were conducted using natural rocks of different sizes, $<0.5 \mathrm{~m}^{3}$, and steel reinforced concrete cubes, with dimensions of 0.42 and $1.0 \mathrm{~m}^{3}$, dropped into a slope, with a height potential of $60 \mathrm{~m}$, leading to impact with the attenuator netting.

The preliminary tests were documented with high speed cameras and load cells measuring the forces in the support ropes. The videos were analysed to determine changes in the rock's kinetic energy, and the deflection of the attenuator net. The objectives of the tests were to find how to optimise system designs to minimise the impact energy absorbed by the netting and support structure. The maximum impact energy achieved during the preliminary test series was in the order of $600 \mathrm{~kJ}$. Following these initial tests, a second test series was performed in January 2016. Additional to the high speed video and load cell measurements, some experiments involved a novel rock motion logger integrated into the blocks centre of mass. The rock motion logger permitted the rocks accelerations and rotations to be captured during contact with the netting. For the first time, measurements of this kind have been made for attenuators and offer detailed insights into the mechanics of the attenuation process.

Further work, in the development of rockfall attenuator systems, is to analyse existing systems, which have been impacted by natural rocks, gaining an estimate of their performance. Alongside these observations, the experimental data is being analysed in detail to refine a new design concept of attenuator barriers. Further results will be given in this paper.
\end{abstract}

\section{Introduction}

The principal function of rockfall attenuator systems is to attenuate the intensity of rockfalls, so that the rocks can pass through the attenuator system, reducing the energy which then is manageable for a down slope catchment area. Such a catchment area can be a catch ditch, a rockfall barrier or an additional attenuator in sequence (Figure 1). 


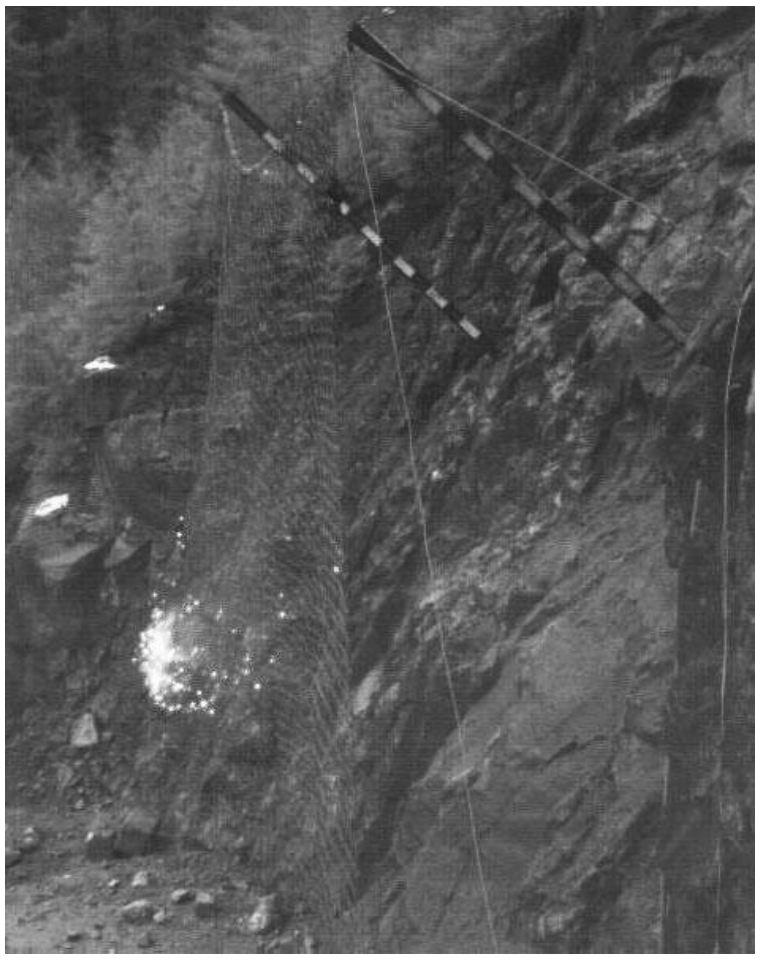

Figure 1 Impact of a natural rock into the attenuator netting. The edges of the rock are caught in the mesh openings causing the rock to jerk and therefore reducing its energy (attenuating)

One of the key differences to standard rockfall barrier system, which catch and contain the entirety of a rock's kinetic energy, is that rockfall attenuator system, deflect and redirect the rock back to the ground, where increased energy losses can occur. In this respect, an attenuator's netting and super structure functions in close conjunction with the terrain. Ideally the main work in attenuating the rocks' kinetic energy is performed through rock-ground contacts and the loading to the attenuator system is kept to a minimum. This is mainly achieved by the attenuator system redirecting the rock's trajectory to the ground. To this end, the concept of rockfall attenuator systems offer the potential to deal with high energy rockfalls with more efficient systems and require lower maintenance than that of standard rockfall barriers. The very nature of rockfall attenuator systems involves a multi-dynamic process of attenuating the rocks kinetic energy. Some of the principal sources of energy losses are achieved through the following interactions:

- Net and rock impacts and frictional contact.

- Rock and ground impacts and frictional contact.

- Net to ground contact friction.

With such a range of possible interactions, providing a clear and quantifiable dimensioning concept for rockfall attenuator systems remains an ongoing research and development task for the rockfall community. To date, although rockfall attenuator systems have been widely applied as a rockfall mitigation solution, their design has been based on much engineering judgement and past experience of a trial an error approach. There still remains a strong need to quantify the behaviour of rockfall attenuator systems during an impact so that a design concept can be produced.

Addressing this need, Geobrugg AG of Switzerland and Wyllie \& Norrish Rock Engineers Ltd. of Canada has embarked upon an extensive research and development campaign designed at documenting rockfall attenuator behaviour and defining the attenuation process. Importantly, this has involved quantifying the complex multi-dynamic mechanics that characterise energy dissipation in rockfall attenuator systems. With this in mind, the design and execution of full scale rockfall testing on rockfall attenuator systems demands a novel approach to testing, involving advanced instrumentation designed at capturing this behaviour. 
Rockfall barrier testing has long been standardised to provide a platform for both a high level research and an international testing standard for conventional rockfall barriers. The first standard for rockfall barrier testing was established by the Swiss Federal Institute for Forest and Landscape Research (WSL), in collaboration with Geobrugg AG, Switzerland. The testing procedure involves an impact of a rock into a rockfall barrier generated from a rock in free fall. This led to the Swiss standard for rockfall barrier testing (Gerber 2001), which was later adapted to fulfil a European standard, the ETAG 027 (EOTA 2008).

For standard rockfall barriers that are designed to absorb the entirety of a rock's kinetic energy, the aforementioned testing technique is fairly straightforward to perform. This is because, with a free fall load case, the design energy of the rockfall barrier can easily be prescribed in the test design by altering the mass of the rock and the free fall height. However, for rockfall attenuators, this testing approach is not applicable due to their mechanics being closely coupled to interactions with the ground, not least that their design permits passage of the rock through the netting system.

Developing a testing technique for rockfall attenuator systems is particularly challenging due to the difficulties in accounting for all the sources of potential energy loss from the rock as it passes through the system. Moreover, because rockfall attenuator systems are designed to be placed in slope, the load case must also impact the netting in slope, which rules out the possibility for a free fall impact. As a result, rocks must be rolled into the slope to insure the correct impact. Rockfalls are inherently random during rebound, which inevitably makes the task of producing repeatable tests extremely difficult. As a result of this, the testing procedure demands that a number of tests are performed, in which the attenuator system is impacted by rocks, so a representative array of potential impact cases can be measured.

To meet these challenges, the testing approach at the rockfall barrier test site at Nicolum quarry in Hope, British Columbia (BC) involved a novel testing procedure along with state-of-the-art measurement techniques and energy budget accounting.

\section{$2 \quad$ Testing approach, apparatus and observations}

The purpose of this paper is to illustrate how the rockfall attenuator test site has been designed to capture the complex mechanics of rockfall attenuator behaviour. The following section provides an overview of the steps and techniques applied in the research and development program for attenuator systems. To provide an applied insight into the advanced capabilities of the Hope BC rockfall testing facility, a single experiment from the experimental campaign is used to illustrate how each analysis technique is performed.

In all, over 80 rockfall experiments were conducted during the most recent testing series in January 2016. The data of these experiments are currently being analysed in detail to consolidate the current advances in our understanding of rockfall attenuator systems and towards producing a design concept. Furthermore, different steel wire netting geometries, others supporting structures or other supporting ropes setup, could be tested.

\section{$2.1 \quad$ Test slope}

The test site in Hope, $\mathrm{BC}$, comprises of a rock slope with a potential height of $60 \mathrm{~m}$. The attenuator test barrier was installed towards the lower part of the slope, so that rocks could be rolled onto the slope with the use of an excavator, where the rock slope geometry, is favourable to the block's transition, to the point of impact with the attenuator system (Figure 2). This particular form ensured maximum acceleration of the rock, reducing the potential for severe slope impacts that might retard the rock's velocity. With a free approach in slope rock rolling, it was possible to generate an array of different impact conditions to test the attenuator systems. Rocks impacted the attenuator netting at varying initial angles, i.e. near perpendicular to netting and close to parallel to the netting, in addition to a range of velocities. With this setup, the array of different load cases simulates the likely real-life load cases faced by attenuator systems when used for rockfall mitigation. 


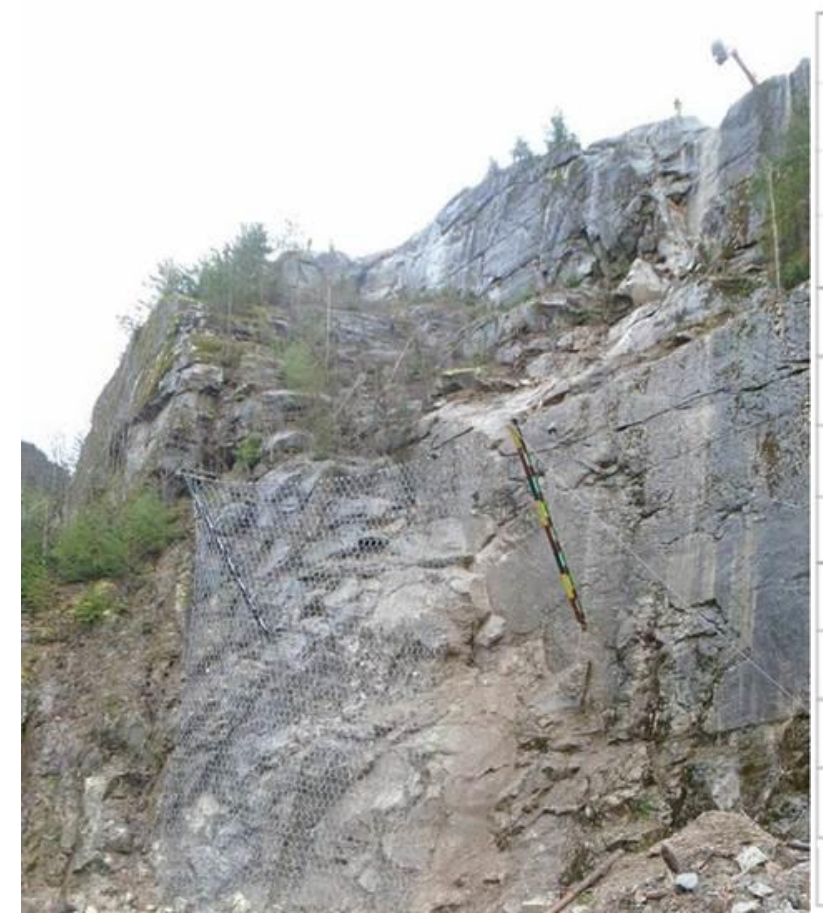

(a)

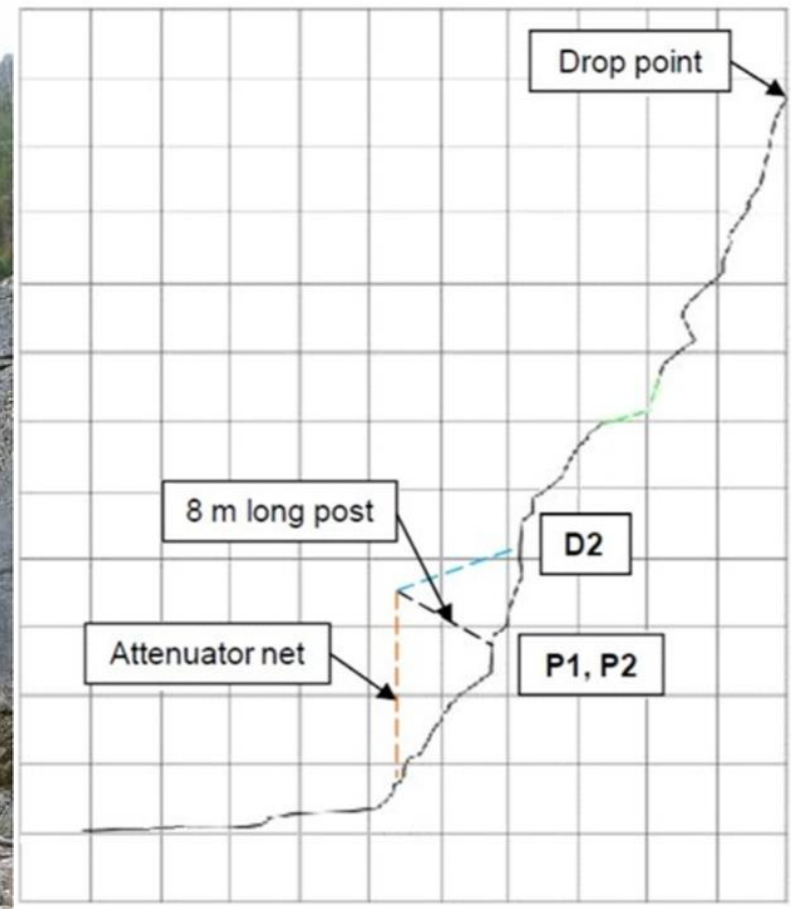

(b)

Figure 2 (a) Image of test slope with release zone at the top from a digger and attenuator netting installed in the lower portion of the slope. (b) Test site slope profile of the test slope with position of the attenuator netting under testing, P1 and P2 are the post position and D2 the location of anchoring

\section{$2.2 \quad$ Test rocks}

Both quarry rocks, selected while onsite, and prefabricated reinforced concrete blocks, were used during testing to impact the attenuator system. Natural rocks were selected between 0.1 and $0.5 \mathrm{~m}^{3}$ while the reinforced concrete blocks were 0.42 and $1.0 \mathrm{~m}^{3}$ in volume (Figure 3). Their weight varied slightly depending on the amount of steel reinforcement used in their construction. Select concrete test blocks were equipped with a central pipe enclosure designed to house the rock motion sensors.

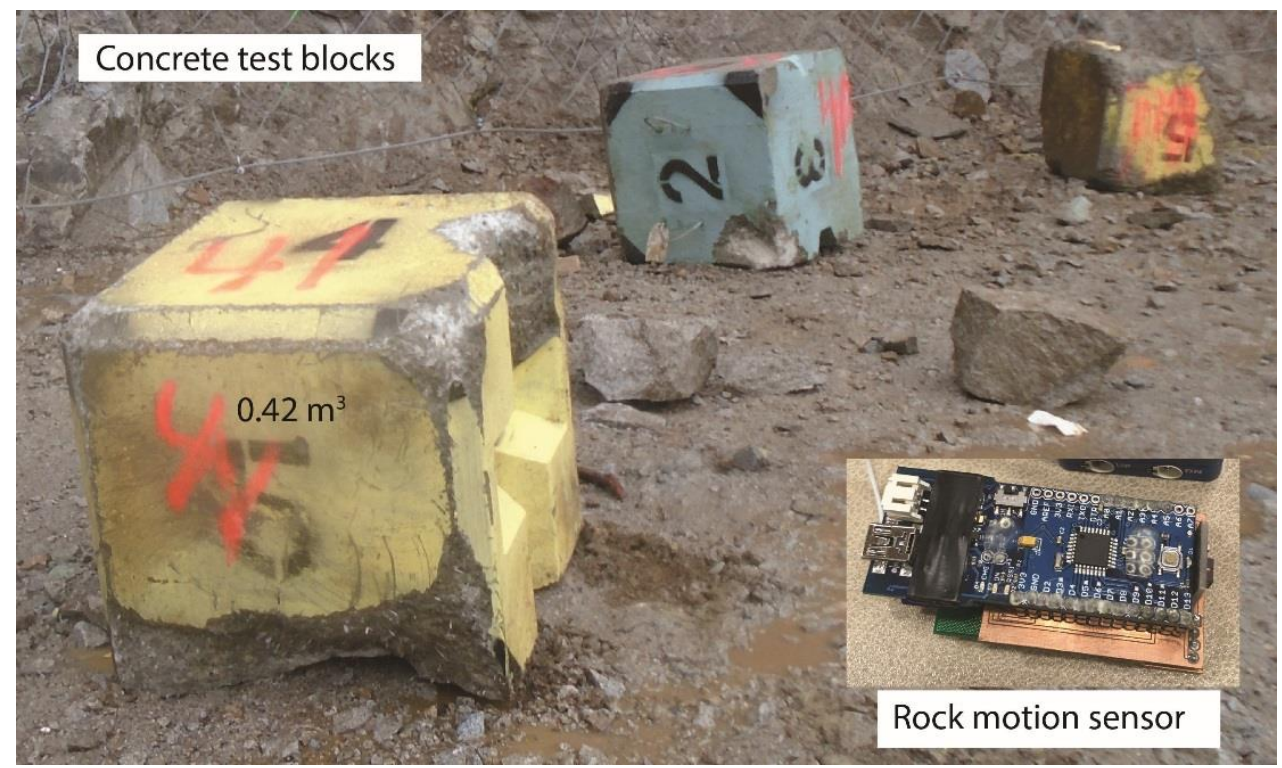

Figure 3 Concrete test blocks retained by the attenuator netting and contained in the catchment area. The rock motion sensor was placed in the centre-of-mass of the blocks and measured the three-dimensional rock accelerations and rotations during the experiments 


\subsection{Video analysis}

Primarily, video analysis is used to extract the velocity of the rocks before impacting the attenuator netting (Figure 4). Additional parameters, such as initial impact angle of the rock into the net and the amount of deflection of the net from its original position, can also be extracted from the video.

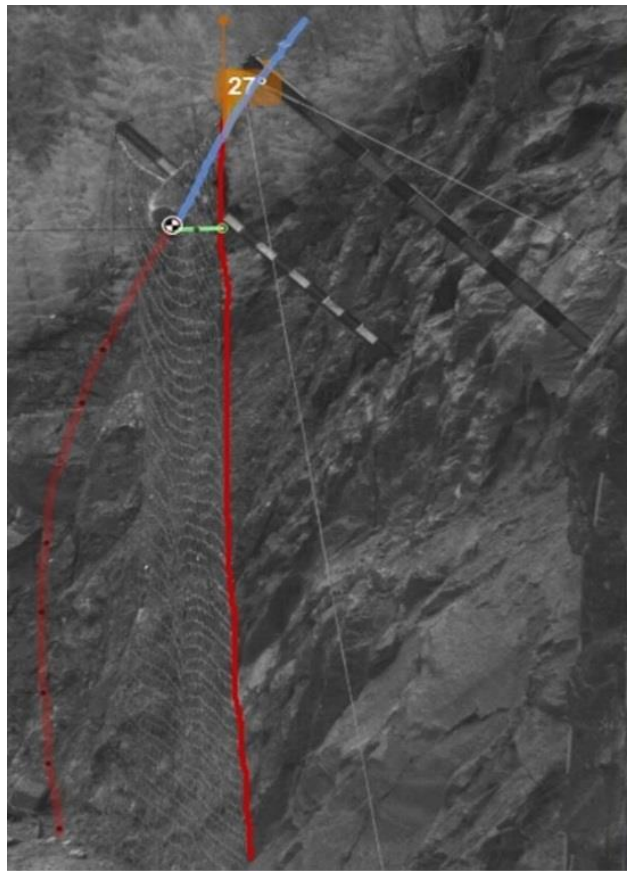

(a)

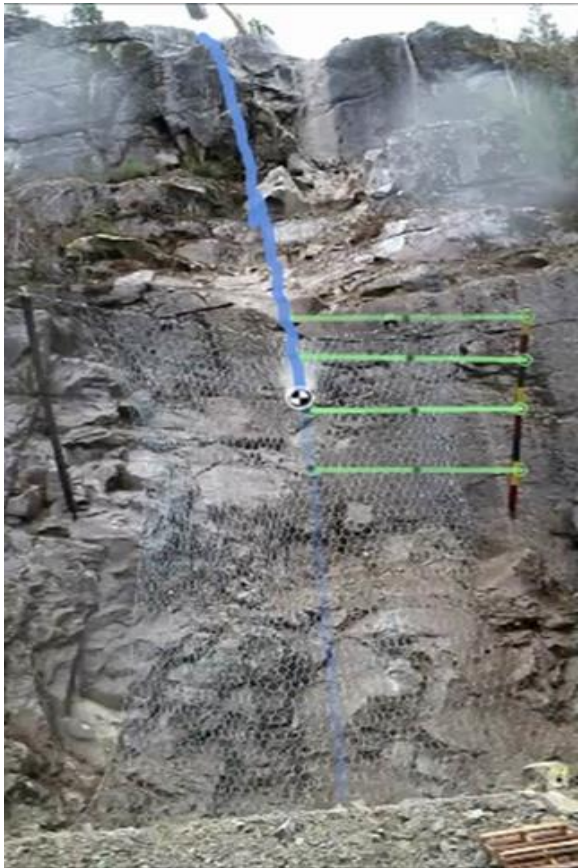

(b)

Figure 4 Video analysis performed using Kinovea motion analysis software. (a) Captures the analysis performed on the side view camera. The angle is measured of the initial impact with the netting. The position of the rock during free fall, and whilst in contact with the net, is plotted over time. Finally the deflection of the net, with respect to its original vertical position, is measured at select points over the trajectory. (b) The frontal camera view documents the lateral distance of the rock to the side view camera by measuring the distance with respect to the scaling bar

The main velocity analysis and observations of the rock-net interaction are performed in two-dimensions using a side view camera. The high speed camera captures the initial impact trajectory and the rock-net interaction in the full field of view. The images are scaled using Kinovea, by assigning pixels in the image a known distance (Figure 4(a)). Following this, the applied scale is then corrected for depth of field variations due to the position of the passing rock in relation to the initial scale bar. The depth of field of the rock is attained using an additional front view camera (Figure 4(b)).

The correction of the applied image scaling varies according to the position of the rock. A moving correction is applied to the corresponding time section of the side view image data. The quality of the video analysis and the image scaling is performed by plotting the vertical component of the free fall trajectory and comparing it to the theoretical free fall position between the two points, if gravitational acceleration is applied. Using the observed velocity and the known mass of the rock the rock's translational kinetic energy $\left(k E_{\text {trans }}\right)$ can be resolved using the equation for translational kinetic energy, wherein $m$ is the body's mass and $v$ its velocity:

$$
k E_{\text {trans }}=\frac{1}{2} m v^{2}
$$

This does not account for the additional kinetic energy the rock holds due to its rotation. The rotational component of the rock's motion can also be extracted from the video analysis, albeit a highly labour intensive task. In this respect, the use of the rock motion sensor provides a direct reading of the rock's angular velocity. Details of the embedded rock motion sensor are provided in the following sections. 


\subsection{Attenuator system and load cells}

For the testing series presented herein the attenuator system was composed of two supporting posts that were anchored into the slope on: hinged base plates, a top support rope, upslope retaining ropes, and lateral support ropes (Figure 2(a)). The attenuator netting was constructed of high-tensile steel wire mesh and could be hung between the two supporting system posts from the top support rope. Using this setup it is possible to interchange different net types with ease. This facilitates an efficient turnaround during testing so that the research and development goals of documenting the behaviour of the different wire mesh designs could be met.

In order to measure the forces that the attenuator system experiences during an impact, all the supporting ropes were instrumented with load cells. The load cells require a data acquisition system to provide an excitement voltage and measure the change in resistance according to the strain under the applied load. Initial calibration experiments, prior to installing the load cells into the attenuator barrier; provide the conversion of strain to force, which is given in kilo Newton's (kN).

The load cell data acquisition system sampled the rope forces at $2.4 \mathrm{kHz}$ and was triggered using a force exceedance threshold. Figure 5 provides an example of typical rope forces during a rockfall experiment.

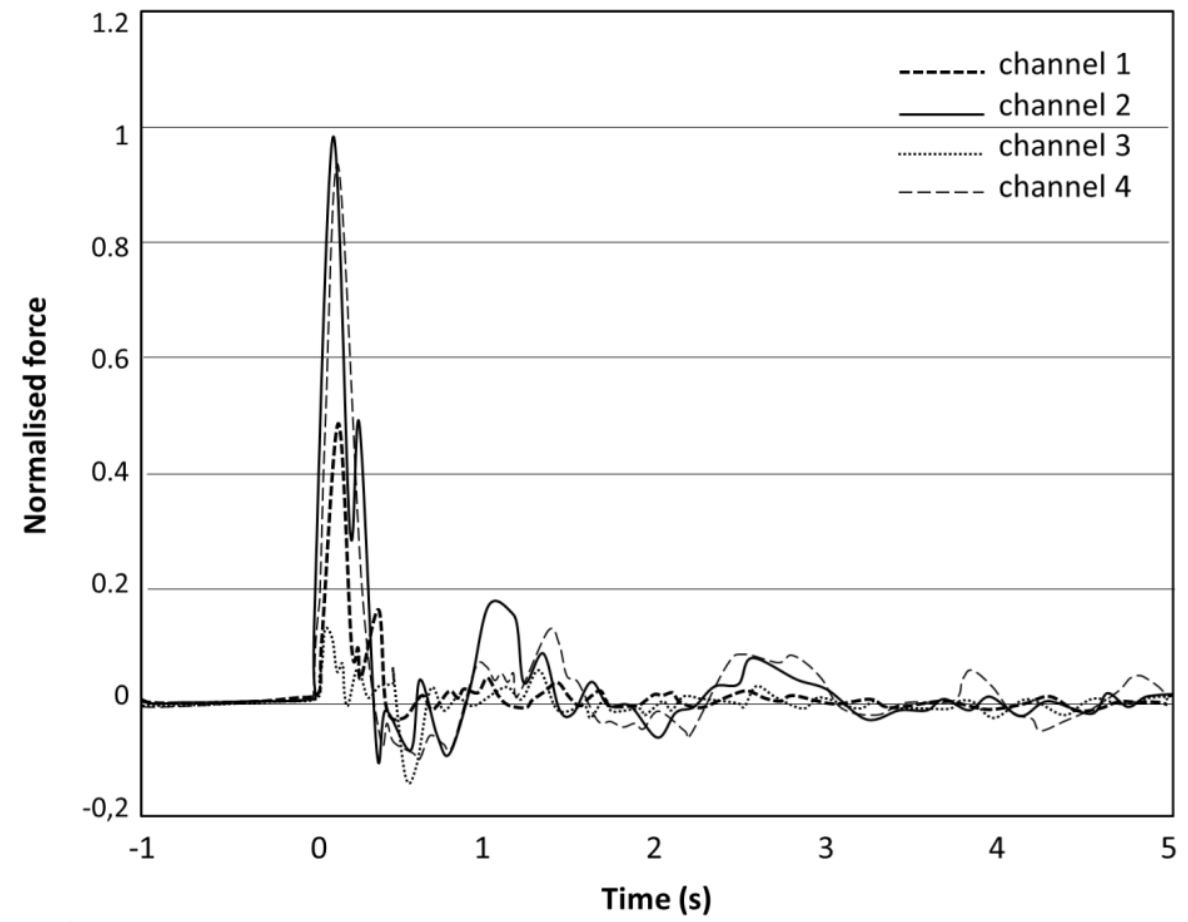

Figure 5 The load cell reading of rockfall impact into the attenuator net and provides force (kN) over time (s) for each of the operating channels in the data acquisition system

\subsection{Rock motion sensor}

In order to capture the full dynamics of the rocks motion, during experimentation, a novel sensor bundle was employed to capture the full three-dimensional accelerations and rotations of the rock. The rock motion sensor is a modular device that can capture acceleration about each principal axis of inertia up to a maximum of $500 \mathrm{~g}$ and rotations up to an angular rate of 18,000 degrees per second. The sampling rate of the sensor is $20 \mathrm{kHz}$.

\subsubsection{Accelerometers}

The operational range of the accelerometers is from $1 \mathrm{~g}$ up to $500 \mathrm{~g}$ with a noise level of $0.5 \mathrm{~g}$. This particular sensor, was selected because it could cope the strong contrast, in the expected accelerations 
during experimentation. For rock on rock impacts the accelerations are severe and the upper limit of $500 \mathrm{~g}$ serves to capture such impacts. On the other hand, once the rock enters the more flexible steel wire netting of the attenuator system we are interested in the decelerations the rock experiences as it comes into contact with the much softer surface of the netting. In this case, we anticipate maximum decelerations in the order of $10.0 \mathrm{~g}$. For this to be possible, the noise floor of the sensor should be kept to a minimum so that both low and high end accelerations can be captured.

\subsubsection{Rock rotations - gyroscope}

The gyroscopes fitted in the rock motion sensor module measure angular rate of change $\omega$ and are capable of measuring 18,000 degrees per second about each principal inertial axis, which is respectively 50 rotations per second or in radians $314 \mathrm{rad}^{-1} \mathrm{~s}^{-1}$. The noise floor of the gyroscopes is around 20 degrees per second. With such a range of rotational velocity, the sensor is suitable for a wide range of different rock sizes. Often the highest rotational velocities are observed with the smaller rocks.

As an example, Figure 6 plots the proportion of the maximum rotational kinetic energy for each principal axis and the Euclidean norm of the rotations experienced during the experiments. The rotational kinetic is defined by the mass of the rock $(m)$ rotating about the inertial axis, and is given in Equation (2) wherein $I$ is the body's moment of inertia and $\omega$ is its rotational speed:

$$
k E_{\text {rot }}=\frac{1}{2} I \omega^{2}
$$

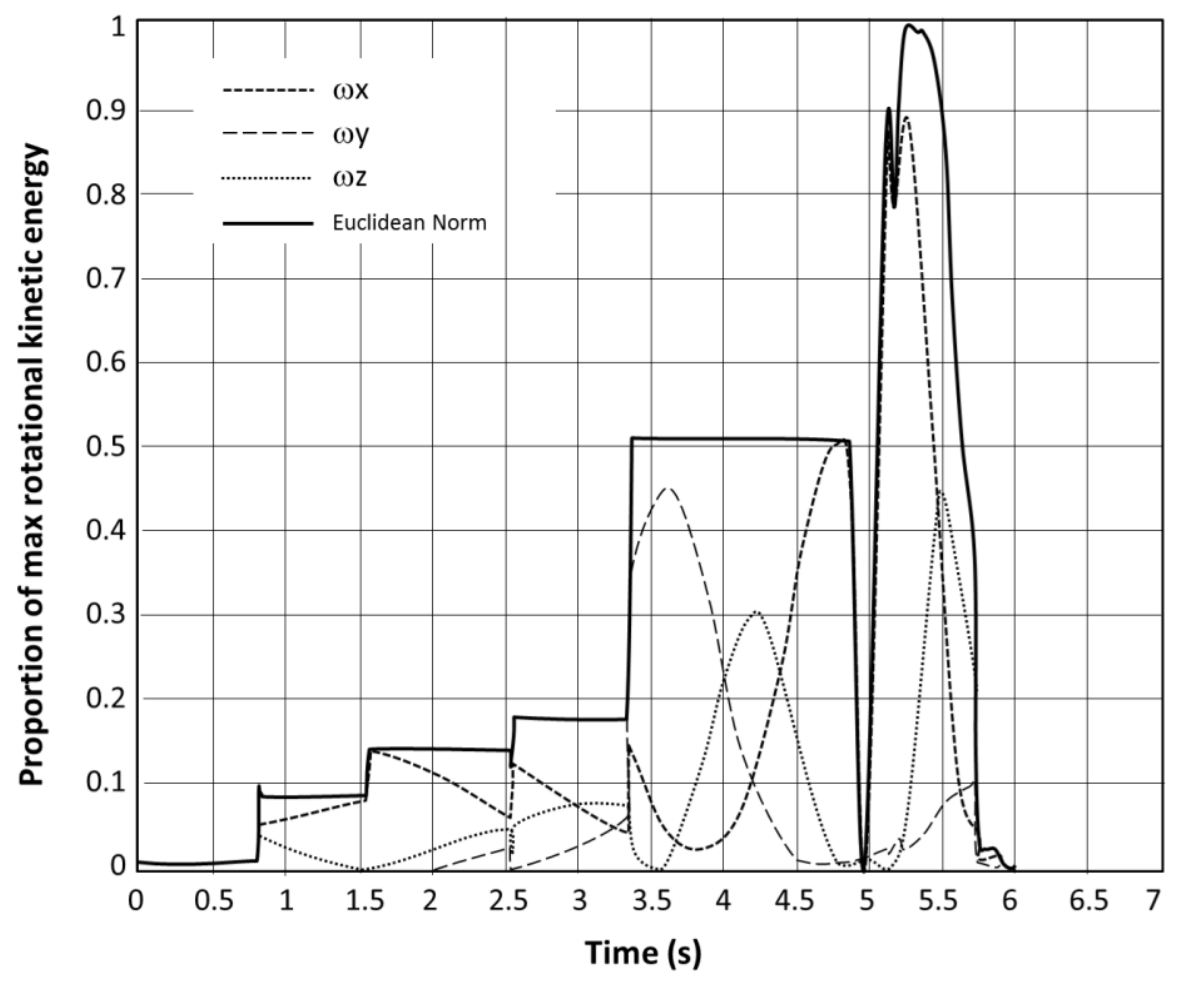

Figure 6 Plot of the proportion of rotational kinetic energy of the rocks, with respect to the maximum rotational kinetic energy. The Euclidean norm is represented as an absolute value, and the proportion for each principal axis of inertia are also plotted

\section{Discussion and conclusion}

With this new testing procedure and measurement technique it is now possible to capture the full dynamics of rockfall and characteristic behaviour of rockfall attenuators. With the addition of the rock motion sensor measuring the rocks accelerations and rotation in three-dimensions the past standard rockfall barrier testing standard is complimented and a fuller account of the rock's energy budget can be 
made. Furthermore, greater insights into the complex interactions of the rock and net can be made detailing the mechanisms with which attenuation of a rocks energy occur.

In addition to the rockfall impact energy budget, with the rock motion sensor it is now possible to measure the rotational kinetic energy of the rock before impact with the net. To date, this was only possible through laborious video analysis techniques, which as a result, were often neglected. Not least, is the essential information to define the initial impact condition of the rock into the net, also this test results include some of the first measurements with which the relationship of energy transfer, between translational and rotational kinetic energy, that occurs during rockfall rebounds on terrain. Understanding these mechanisms in rockfall is an area of ongoing research for rockfall science.

Hereinafter, to define the impact case, the rock motion sensor is allowing a more complete examination of how attenuator systems function. From the acceleration data, it is possible to observe how the rock is decelerated due to the impulse of the netting, and this coupled with the force readings from the load cells, the cause and affect coupling of rock impact and system reaction can be studied. Importantly for a dimensioning concept for rockfall attenuator systems, this will help to define the maximum possible impact an attenuator system design can withstand. To date, this has remained an objective parameter in attenuator design and in the most part has been selected on engineering judgment as opposed to a calculated and tested value.

In all, this collaborative research and development programme for rockfall barriers and attenuators between Wyllie \& Norrish Rock Engineers Ltd., Canada and Geobrugg North America has facilitated the establishment of a state-of-the-art rockfall testing facility at the Nicolum Quarry in Hope, BC. The testing facility takes the long established experience in rockfall barrier testing of Geobrugg North America and adapts the methodology to suit the complex load case and measurement requirements of testing rockfall attenuator systems. The test site enables correct impact loading of rockfall attenuator systems, as would be expected in a real-life rockfall attenuator installation. The quarry setting permits testing to be carried out with site machinery, which allows for a high frequency of rockfall tests in a short space of time. In this way it is possible to capture the array of possible load cases (i.e. impact angles, energies and rotations) that is to be anticipated for natural rockfall. Finally, the advanced and novel measurement technique includes video analysis, load cell measurements, and rock motion sensing with use of accelerometers and gyroscopes. These coupled measurements permit a full accountancy of the attenuator energy budget during the attenuation process. To date, a high number of tests could be performed and documented at this testing facility. This data assists in furthering the understanding of rockfall attenuator systems and lays the foundations for a full dimensioning concept.

\section{Acknowledgement}

Wyllie \& Norrish Rock Engineers Ltd. and Geobrugg North America would like to thank all parties who assisted in the successful construction of the rockfall testing facility and also performing the rockfall tests. Special thanks go to Emil Anderson Construction, to both Doug Brown and Rick Bergstrom and their expert crews in getting the rocks to roll into the attenuator systems.

\section{References}

EOTA (European Organisation for Technical Assessment) 2008, Guidelines for European technical approval of falling rock protection kits (ETAG 027), European Organisation for Technical Assessment, Brussels.

Gerber, W 2001, Guideline for the approval of rockfall protection kits, Swiss Agency for Environment, Forest and Landscape (SAEFL) and Swiss Federal Research Institute (WSL), Switzerland.

Glover, J, Denk, M, Bourrier, F, Volkwein, A \& Gerber, W 2012, 'Measuring the kinetic energy dissipation effects of rock fall attenuating systems with video analysis', in 12th Congress INTERPRAEVENT, vol. 1, pp. 151-160.

Kinovea Motion Analysis Software 2016, Informer Technologies, Inc. 